\title{
Accurate micro Hall effect measurements on scribe line pads
}

Østerberg, Frederik Westergaard; Petersen, Dirch Hjorth; Wang, Fei; Rosseel, E.; Vandervorst, W.; Hansen, Ole

Published in:

17th IEEE International conference on Advanced Thermal Processing of Semiconductors, RTP09

Link to article, DOI:

10.1109/RTP.2009.5373450

Publication date:

2009

Document Version

Publisher's PDF, also known as Version of record

Link back to DTU Orbit

Citation (APA):

Østerberg, F. W., Petersen, D. H., Wang, F., Rosseel, E., Vandervorst, W., \& Hansen, O. (2009). Accurate micro Hall effect measurements on scribe line pads. In 17th IEEE International conference on Advanced Thermal Processing of Semiconductors, RTP09 (pp. 157-162). IEEE. https://doi.org/10.1109/RTP.2009.5373450

\section{General rights}

Copyright and moral rights for the publications made accessible in the public portal are retained by the authors and/or other copyright owners and it is a condition of accessing publications that users recognise and abide by the legal requirements associated with these rights.

- Users may download and print one copy of any publication from the public portal for the purpose of private study or research.

- You may not further distribute the material or use it for any profit-making activity or commercial gain

- You may freely distribute the URL identifying the publication in the public portal 
$17^{\text {th }}$ IEEE International Conference on Advanced Thermal Processing of Semiconductors - RTP 2009

\title{
Accurate micro Hall Effect measurements on scribe line pads
}

\author{
F. W. Osterberg ${ }^{a}$, D.H. Petersen ${ }^{a, b}$, F. Wang ${ }^{a}$, E. Rosseel ${ }^{c}$ and W. Vandervorst ${ }^{d, e}$ O. Hansen $^{a, d}$ \\ ${ }^{a}$ DTU Nanotech - Dept. of Micro and Nanotechnology, Technical University of Denmark, \\ building 345 East, DK-2800 Kgs. Lyngby, Denmark \\ ${ }^{b}$ CAPRES A/S, Scion-DTU, building 373, DK-2800 Kgs. Lyngby, Denmark \\ ${ }^{c}$ IMEC, Kapeldreef 75, B-3001 Leuven, Belgium \\ ${ }^{d}$ CINF - Centre for Individual Nanoparticle Functionality, Technical University of Denmark, \\ building 345 East, DK-2800 Kgs. Lyngby, Denmark \\ ${ }^{e}$ Inst. voor Kern- en Stralingsfysica, K. U. Leuven, Celestijnenlaan 200D B-3001 Leuven, Belgium
}

\begin{abstract}
Hall mobility and sheet carrier density are important parameters to monitor in advanced semiconductor production. If micro Hall Effect measurements are done on small pads in scribe lines, these parameters may be measured without using valuable test wafers. We report how Hall mobility can be extracted from micro four-point measurements performed on a rectangular pad. The dimension of the investigated pad is $400 \times 430 \mu^{2}$, and the probe pitches range from $20 \mu \mathrm{m}$ to $50 \mu \mathrm{m}$. The Monte Carlo method is used to find the optimal way to perform the Hall measurement and extract Hall mobility most accurate in less than a minute. Measurements are performed on shallow trench isolation patterned silicon wafers to verify the results from the Monte Carlo method.
\end{abstract}

\section{INTRODUCTION}

In processing of semiconductor devices, it is becoming increasingly important and difficult to be able to characterize material parameters such as sheet resistance, sheet carrier density and Hall mobility. These parameters can be characterized through various methods, where some require special sample preparation, and others are destructive [1], [2]. It has previously been shown that micro four-point probes are able to measure sheet resistance, sheet carrier density and Hall mobility on thin film samples with at least one insulating boundary [3].

The existing technique has been validated experimentally for samples with a single boundary, while assuming a uniform sheet resistance throughout the entire sample, which allows for position error suppression thus giving very accurate results [4].

In this paper we investigate the possibility of performing micro Hall Effect measurements in less than a minute on small $\left(400 \times 430 \mu^{2}\right)$ test pads, which do not necessarily have uniform sheet resistance. Being able to extract sheet resistance, sheet carrier density and Hall mobility from small samples is a significant advantage, as these can be patterned in scribe lines on the actual wafer, thus reducing the need for expensive test wafers.

\section{THEORY}

A. Micro Hall Effect measurements: Micro Hall Effect measurements are performed on thin film samples using a micro four-point probe. The four electrodes are placed in-line with equal distance between the probes, also called the probe pitch,

987-1-4244-3815-0/09/\$25.00 (C) IEEE
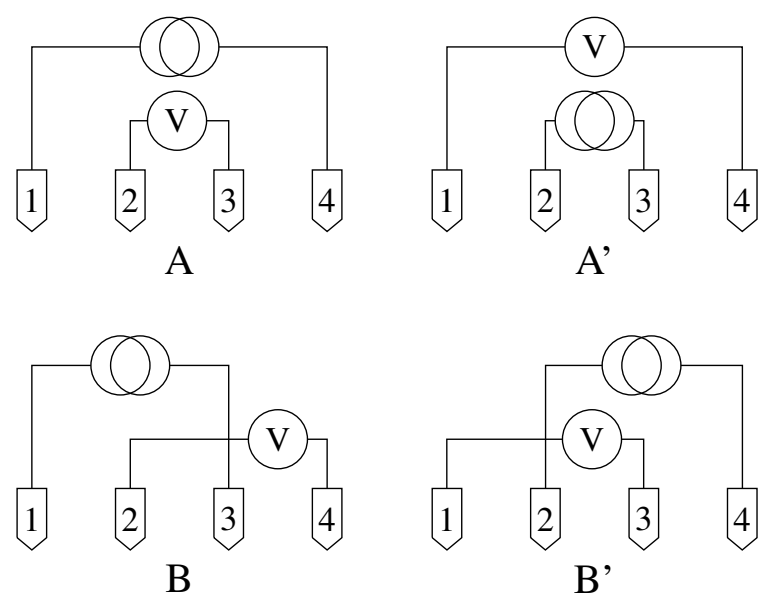

Fig. 1. The four different probe configurations (A, A', B, B') used for Hall Effect measurements.

$s$. An insulating boundary must be present and a magnetic field, $B_{z}$, applied perpendicular to the surface. The actual measurement is done by forcing a current, $I$, through two of the probes pins while measuring the potential difference, $V$, between the two remaining pins. Four different pin configurations (A, A', B, B') are used, and these are illustrated in Fig. 1.

Previous work [3] has shown the convenience of defining the resistance difference as $\Delta R_{B B^{\prime}} \equiv R_{B}-R_{B^{\prime}}$ and the mean resistance as $\overline{R_{B B^{\prime}}} \equiv\left(R_{B}+R_{B^{\prime}}\right) / 2$, where $R_{B}$ and $R_{B^{\prime}}$ are the resistances, $R=V / I$ measured in configurations $\mathrm{B}$ and $\mathrm{B}$ ' respectively, similar values can be computed for the A and A' configurations. Furthermore, it has been shown that introducing a pseudo sheet resistance, $R_{P}$, greatly reduces position errors [4]:

$$
\exp \frac{2 \pi \overline{R_{A A^{\prime}}}}{R_{P}}-\exp \frac{2 \pi \overline{R_{B B^{\prime}}}}{R_{P}}=1 .
$$

Simple analytical expressions can be derived for $\Delta R_{B B^{\prime}}$, $\overline{R_{B B^{\prime}}}$ and $\overline{R_{A A^{\prime}}}$ for homogeneous thin films with geometries such as the infinite half plane. Since (1) cannot be solved analytically for $R_{P}$, it is solved numerically. What makes $R_{P}$ and $\Delta R_{B B^{\prime}}$ convenient is the fact that they are proportional 
to the sheet resistance $R_{0}$ and the Hall sheet resistance $R_{H}$, respectively. This allows for expressing them in the following manner:

$$
\begin{gathered}
R_{P}=R_{0} f\left(\frac{y}{s}\right), \\
\Delta R_{B B^{\prime}}=R_{H} g\left(\frac{y}{s}\right),
\end{gathered}
$$

where $f(x)$ and $g(x)$ are theoretically calculated functions and $y$ is the distance to the barrier. When measurements are fitted to (2) and (3) to give $R_{0}$ and $R_{H}$, they can be used to calculate the Hall mobility $\mu_{H}$ :

$$
\mu_{H}=\frac{Z R_{H}}{R_{0} B_{z}}
$$

where $Z$ is the sign of the carrier charge.

B. Measurement strategy: A procedure for extracting Hall mobility from measurements performed on homogeneous thin films has previously been described [4]. In short, this method dictates that two measurements should be performed with e.g. a $20 \mu \mathrm{m}$ pitch probe; one roughly $4 \mu \mathrm{m}$ from the boundary and the other $60 \mu \mathrm{m}$ from the boundary. The measurements are then fitted to the theoretical expressions for the infinite half plane. This method can be performed in less than a minute, and will therefore be referred to as the "Fast - $3 \mathrm{~s}$ apart" (F3S) method. The F3S method can also be applied to measurements on other geometries as long as the theoretical values used for fitting are those for the given geometry. Since a $400 \times 430 \mu^{2}$ rectangle cannot be treated as an infinite half-plane the theoretical values used for fitting are computed using numerically conformal mapping [5]. A potential flaw with this method is that it assumes uniform sheet resistance for the sample and in particular at the two points measured. Since practical samples often have nonuniform sheet resistance a new measurement strategy will be proposed.

1) New measurement strategy: Here another fast method will be proposed, called the "Fast - with Small Separation" (FSS) method. The idea behind the new FSS method is that despite the degree of sheet resistance variation, it can be assumed constant within a significant small area. This will allow for performing two measurements with a small separation and then fitting to the theoretical expressions for a constant sheet resistance. In the following the method will be derived.

Two measurements are to be performed; the first measurement point is placed $y_{0}$ from the boundary and the second $\Delta y$ further away. However, both values are assumed unknown due to the uncertainties on the actual probe positions. The two measurements will each result in a value for $R_{P}$ and $\Delta R_{B B^{\prime}}$, which leads to four equations with four unknowns:

For the pseudo sheet resistance:

$$
\begin{gathered}
R_{P 1}=R_{0} f\left(\frac{y_{0}}{s}\right), \\
R_{P 2}=R_{0} f\left(\frac{y_{0}+\Delta y}{s}\right),
\end{gathered}
$$

and for the resistance difference:

$$
\begin{gathered}
\Delta R_{B B^{\prime} 1}=R_{H} g\left(\frac{y_{0}}{s}\right), \\
\Delta R_{B B^{\prime} 2}=R_{H} g\left(\frac{y_{0}+\Delta y}{s}\right),
\end{gathered}
$$

where $R_{0}, R_{H}, y_{0}, \Delta y$ are all unknowns.

By eliminating $R_{0}$ and $R_{H}$, two equations with two unknowns are obtained:

$$
\begin{gathered}
\frac{R_{P 1}}{R_{P 2}}=\frac{f\left(\frac{y_{0}}{s}\right)}{f\left(\frac{y_{0}+\Delta y}{s}\right)}, \\
\frac{\Delta R_{B B^{\prime} 1}}{\Delta R_{B B^{\prime} 2}}=\frac{g\left(\frac{y_{0}}{s}\right)}{g\left(\frac{y_{0}+\Delta y}{s}\right)} .
\end{gathered}
$$

The two equations cannot be solved analytically and must instead be fitted numerically by using the least square method. Once $y_{0}$ and $\Delta y$ are found, $R_{0}$ is found by doing least square fit of (5) and (6), while $R_{H}$ is found similarly from (7) and (8).

2) Extensive measurement strategy: As a third method to extract the Hall mobility, the "All Inclusive" (AI) method will be presented. For this both a sheet resistance scan and and Hall Effect scan is required. The sheet resistance scan is used to calculated theoretical values for $\Delta R_{B B^{\prime}}$ and $R_{P}$ by assuming a constant Hall mobility and using the finite element method (FEM). The series of Hall Effect measurements is then fitted to the FEM calculated theoretical values, similar to the two previously described methods.

C. Monte Carlo Simulations: Monte Carlo simulations of the FSS method have been performed to determine where to measure the two points such that the Hall mobility is found most accurately. Monte Carlo simulations have been performed for $400 \times 400 \mu \mathrm{m}^{2}$ samples with both uniform and nonuniform sheet resistance using $20 \mu \mathrm{m}$ electrode pitch.

For the samples with uniform sheet resistance the simulated measurement data were obtained by conformal mapping in the same way as the new theoretical values were calculated, as described in [5].

The simulated data for a sample with nonuniform sheet resistance were obtained by using COMSOL FEM simulations, where the sheet resistance was defined to vary like an even fourth order polynomial in the $y$-direction, fitted to experimental measurement, such that the sheet resistance at the boundaries was $75 \%$ of the value at the centre. As sheet resistance is typically most strongly dependent on carrier concentration, the mobility has been assumed constant.

For the Monte Carlo simulations a normal distributed position error has been applied for each probe pin (in-line $\sigma_{x}=$ $0.5 \mu \mathrm{m}$, out-of-line $\sigma_{y}=0.1 \mu \mathrm{m}$ ). Also a normal distributed error on the boundary position was applied $\left(\sigma_{b}=0.1 \mu \mathrm{m}\right)$, and finally a normal distributed electrical error on the resistance was added $\left[\sigma_{R}=R_{0} /\left(1.5 \times 10^{5}\right) \Omega\right] .1000$ simulations are used for each set of $y_{0}$ and $\Delta y$. 


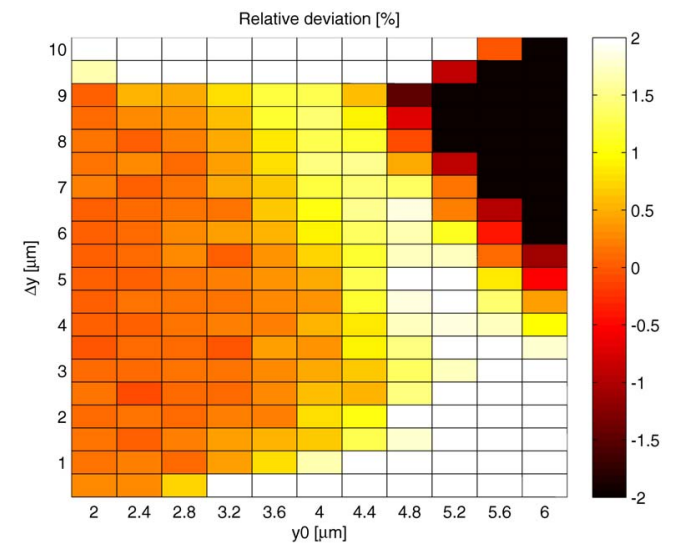

(a)

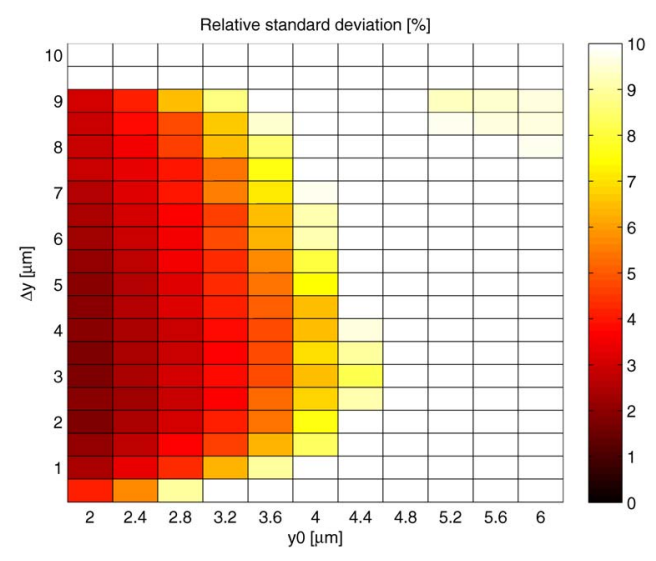

(b)

Fig. 2. Monte Carlo simulations for a sample with uniform sheet resistance, 1000 iterations are used for each point. (a) Contourplot of relative deviation in extracted Hall mobility. (b) Contourplot of relative standard deviation in extracted Hall mobility.

The results of the Monte Carlo simulations for the homogeneous sample is shown in Figs. 2(a) and 2(b). Fig. 2(a) shows the relative deviation of the calculated Hall mobility from the true Hall mobility. It is seen that in order to get correct results the measurements should be performed with $y_{0}$ as small as possible and $\Delta y$ between 1 and $9 \mu \mathrm{m}$. In Fig. 2(b) the relative standard deviation is shown as a function of $y_{0}$ and $\Delta y$. It is seen that $y_{0}$ again should be as small as possible and $\Delta y$ should be around $3 \mu \mathrm{m}$ in order to minimize the relative standard deviation.

In Figs. 3(a) and 3(b), the results from the Monte Carlo simulation on the inhomogeneous sample are shown. From these results it is more difficult to choose which parameters are the optimal to use for real measurements, since the relative deviation is always negative for the chosen variation, which means that the Hall mobility will be estimated too low. To get the best possible value, $\Delta y$ should be as small as possible, but on the other hand a small $\Delta y$ results in a large relative standard deviation which is undesirable.

Even though the results of the Monte Carlo simulation

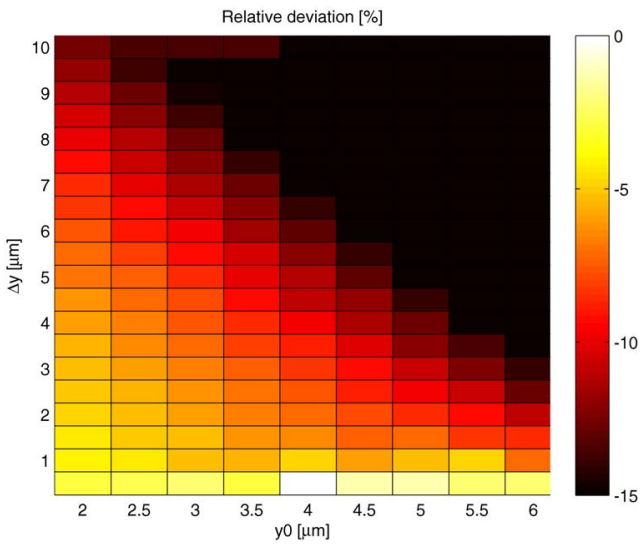

(a)

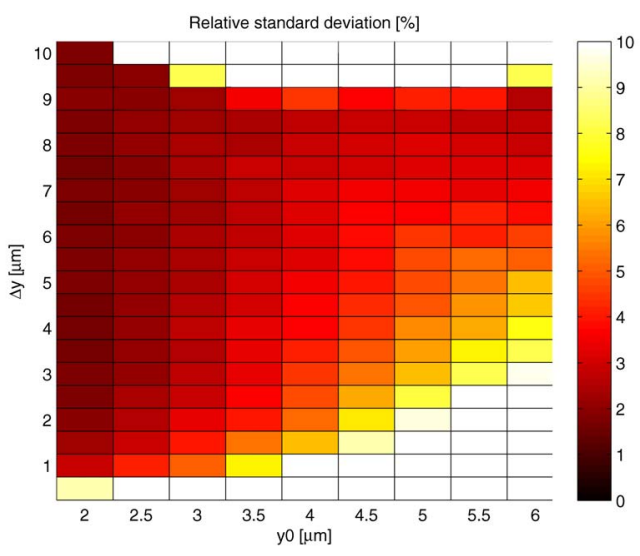

(b)

Fig. 3. Monte Carlo simulations for a sample with nonuniform sheet resistance, 1000 iterations are used for each point. (a) Contourplot of relative deviation in Hall mobility. (b) Contourplot of relative standard deviation in Hall mobility.

indicate that different measurement procedures should be applied for homogeneous and inhomogeneous samples, it has been decided to choose one set of $\Delta y$ and $y_{0}$ to use for all measurements. The reason for this is because when the measurement technique is to be applied, it is unknown whether the sample is completely homogeneous or how inhomogeneous it is. Additional measurements would be required to determine the homogeneity of the sample before performing the actual Hall Effect measurement. However, this would be more time consuming than the actual Hall measurement. The values of $\Delta y$ and $y_{0}$ that will be used when performing the measurements are $y_{0} \approx 4 \mu \mathrm{m}$ and $\Delta y=3 \mu \mathrm{m}$ for a probe pitch of $20 \mu \mathrm{m}$.

\section{EXPERIMENTAL}

Measurements have been performed on two different shallow trench isolation (STI) patterned silicon wafers. Both are implanted at $0.5 \mathrm{keV}$ with a nominal dose of $1 \times 10^{15} \mathrm{~cm}^{-2}$ $\mathrm{B}$, and laser annealed in an Applied Materials DSA chamber [6] at a nominal temperature of $\sim 1200^{\circ} \mathrm{C}$. The difference 
between the two wafers is that on one of them a $400 \mathrm{~nm}$ thick absorber layer (AL) was deposited after the B implant, and stripped again after the laser anneal in an oxygen plasma asher. Measurements have been performed on $400 \times 430 \mu \mathrm{m}^{2}$ pads on both wafers. The pads have been chosen with the same coordinates on both wafers to ensure that they have had similar preparation, except for the AL.

For the measurements a CAPRES microRSP-M150 system [7] has been used. For the Hall Effect measurement a permanent magnet was placed at the centre of the sample chuck. The magnetic flux density at the position of measurements was measured to $0.50 \mathrm{~T}$ using a calibrated Hall sensor. All the M4PP used for measurements had a strain gauge positioned next to the probe, to allow for accurate surface detection.

A. Sheet resistance scans: From the theory it is clear that the uniformity of the sheet resistance is very important when Hall mobility is to be extracted from the Hall measurements. For this reason, sheet resistance scans have been performed on the pads used for Hall measurements.

The sheet resistance scans were performed by scanning at the centre of the pad in the direction parallel to the probe pins, thus eliminating geometrical errors in proximity of the pad edges [8]. The scans are composed of two separate scans. One measured in a dense scan close to the boundary $(<50 \mu \mathrm{m})$ with a probe pitch of $1.5 \mu \mathrm{m}$ and the other measured away from the boundary $(>30 \mu \mathrm{m})$ using a probe pitch of $10 \mu \mathrm{m}$.

B. Micro Hall Effect measurements: Hall measurements have been performed on the same pads as used for the sheet resistance scans. The three different approaches described in section II-B have all been used to extract the Hall mobility. Hall measurements have been performed at all four sides of the measured squares with three different probe pitches; $s=20 \mu \mathrm{m}, s=30 \mu \mathrm{m}$ and $s=50 \mu \mathrm{m}$.

\section{RESUlts}

A. Sheet resistance scans: The results of the sheet resistance scans of the wafer processed without and with the AL are shown in Figs. 4(a) and 4(b) respectively. The measured sheet resistance is plotted as a function of the distance to the boundary orthogonal to the scan direction, which means that the centre of the pad is $215 \mu \mathrm{m}$ from the boundary when scanning in the west - east direction and only $200 \mu \mathrm{m}$ when scanning in the north - south direction, which is also illustrated in the inset.

\section{B. Micro Hall Effect measurements:}

1) The All Inclusive (AI) method: As it has been shown in Fig. 4(a), the sheet resistance is not uniform on the investigated samples. Since most of the sheet resistance scans are completely different, theoretical values must be computed for each sample before fitting. A set of these fits are shown for the edges north and south in Fig. 5. Here both measured $\Delta R_{B B^{\prime}}$ and $R_{P}$ are plotted as well as the theoretically fitted results. The corresponding sheet resistance scan from Fig. 4(a), used to compute new theoretical values, has also been plotted, and as expected it is seen that this fits the theoretical $R_{P}$ in the range $50-350 \mu \mathrm{m}$.

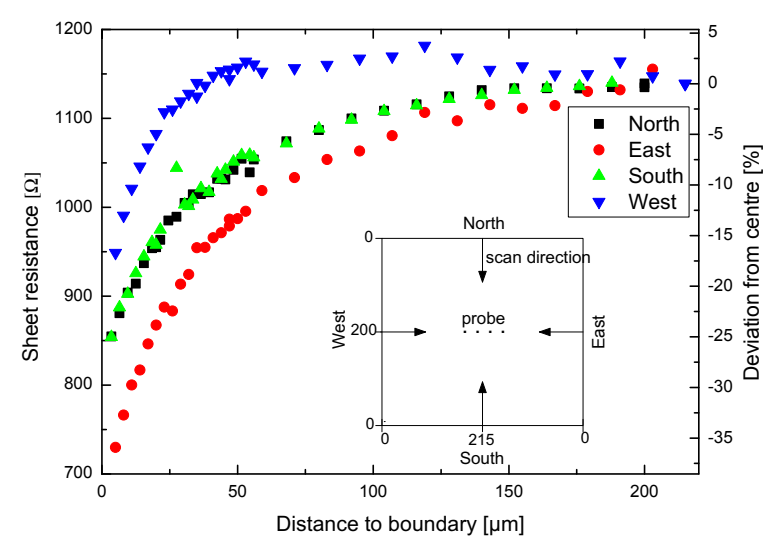

(a)

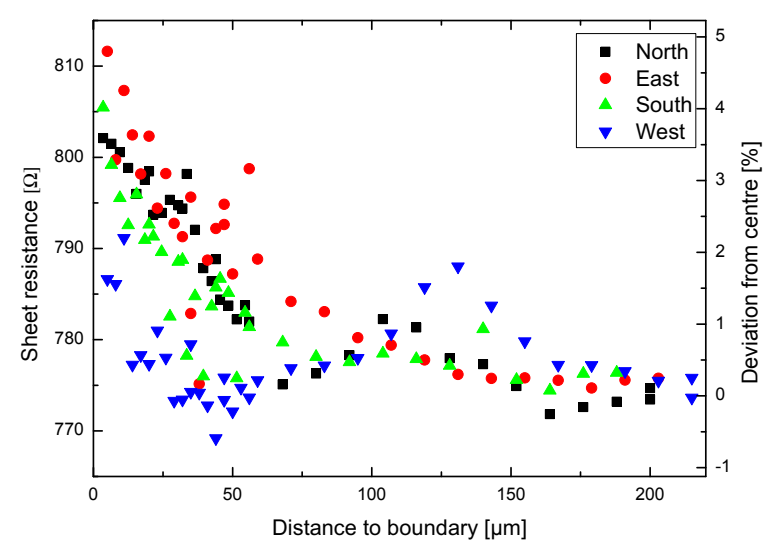

(b) With AL

Fig. 4. Sheet resistance scans on $400 \times 430 \mu \mathrm{m}^{2}$ pads. (a) Measurements on pad processed without AL. The inset shows how the scans are performed, starting with 0 at the boundary and scanning towards the centre, the probe positioned in the middle is aligned in the west - east scan direction, for north - south it would be rotated $90^{\circ}$. (b) Measurements on pad processed with AL.

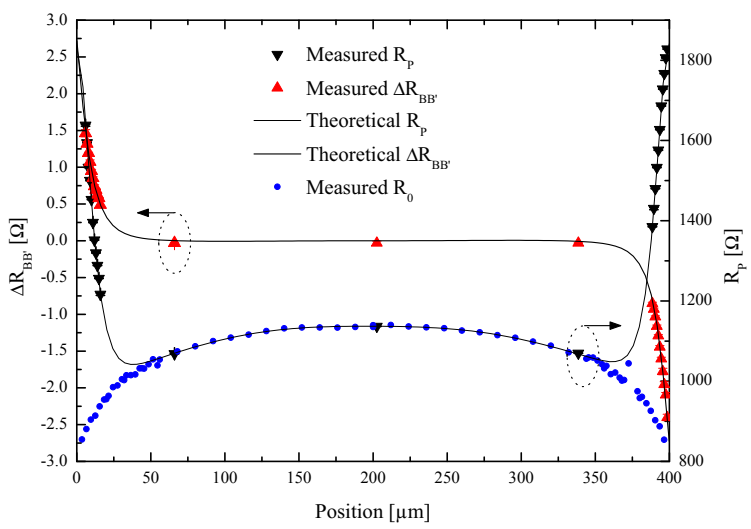

Fig. 5. Fits of theoretical values to sheet resistance and Hall Effect measurement using varying sheet resistance. Measured at north $(400 \mu \mathrm{m})$ and south $(0 \mu \mathrm{m})$ edges on pad processed without AL. The sheet resistance scan from Fig. 4(a) has also been plotted here.

The AI method have been applied for all four edges of the square with the three different probe pitches, resulting in twelve Hall mobility values. As the mobility is assumed to 
TABLE I

MEAN Hall Mobilities EXTRACTEd USING 20, 30 AND $50 \mu \mathrm{M}$ PITCH.

\begin{tabular}{|l|c|c|}
\hline & No AL & With AL \\
\hline & $\overline{\mu_{H}} \pm \sigma_{\mu_{H}}\left[\mathrm{~cm}^{2} \mathrm{~V}^{-1} \mathrm{~s}^{-1}\right]$ & $\overline{\mu_{H}} \pm \sigma_{\mu_{H}}\left[\mathrm{~cm}^{2} \mathrm{~V}^{-1} \mathrm{~s}^{-1}\right]$ \\
\hline AI & $32.4 \pm 0.6$ & $31.4 \pm 0.3$ \\
\hline F3S & $43.2 \pm 4.5$ & $28.5 \pm 1.9$ \\
\hline FSS & $28.3 \pm 1.6$ & $30.6 \pm 1.6$ \\
\hline
\end{tabular}

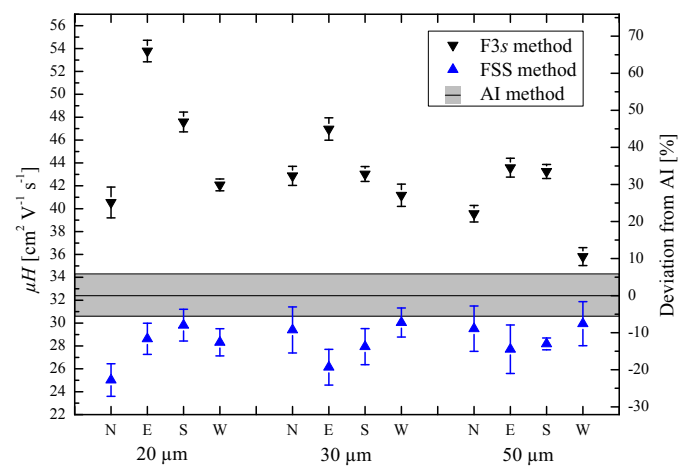

(a)

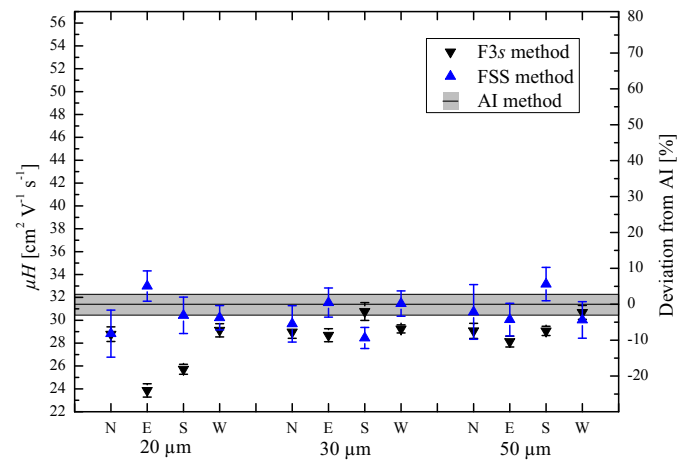

(b)

Fig. 6. Hall mobilities extracted from Hall measurements on 400x430 $\mu \mathrm{m}^{2}$ pads. N, E, S and $\mathrm{W}$ indicates which boundary the measurement is performed at, and the numbers 20,30 and $50 \mu \mathrm{m}$ are the probe pitch used. The grey bands are $\overline{\mu_{H}} \pm 3 \sigma_{\mu_{H}}$ found using the AI method. (a) Sample without AL. (b) sample with AL.

be uniform throughout the pad, the mean Hall mobility, $\overline{\mu_{H}}$, and standard deviation, $\sigma_{\mu_{H}}$, has been found for each pad and reported in Tab. I.

2) The Fast methods: The results of the measurements using the FSS and F3S methods can be seen in Fig. 6. On the same plot the Hall mobility values from the AI method are plotted as grey bands, which corresponds to $\overline{\mu_{H}} \pm 3 \sigma_{\mu_{H}}$. These bands are believed to be the true values as they are found using the most correct theoretical data and all experimental data.

\section{Discussion}

A. Sheet resistance scans: From Figs. 4(a) and 4(b) it is seen that the change of the sheet resistance towards the edge is much larger for the wafer without AL than for the one with
$\mathrm{AL}$. The reason for this is believed to be related to the change in the optical reflection coefficient from oxide covered areas to noncovered areas, giving rise to a significant higher anneal temperature near the oxide edge. This difference is addressed in more detail in [9]. Whereas the sheet resistance decreases by $15-35 \%$ toward the edge on the wafer without the AL, it increases by $2-5 \%$ for the wafer with the AL.

B. Micro Hall Effect measurements: Based on the results from the Monte Carlo simulations of the FSS method, it is expected that the measured Hall mobility will be lower than the real value for the wafer without the AL and slightly higher for the one with the AL.

From Tab. I it is seen that the average Hall mobility extracted by the FSS method on the pad without AL is $13 \%$ below the value found using the AI method, which is in good agreement with the Monte Carlo simulations.

Also from Tab. I it is seen that the average Hall mobility extracted from the F3S method is $33 \%$ higher than the AI method value for the nonuniform pad processed without $\mathrm{AL}$ and the relative standard deviation is $10 \%$. Thus, the results clearly demonstrate that this method is useless for highly nonuniform samples as the results are completely wrong.

The extracted Hall mobilities for the more uniform pad processed with $\mathrm{AL}$ are in general placed closer to the expected value for both two point methods. On average the F3S method underestimates the Hall mobility by $9.2 \%$, whereas the FSS method is just $2.3 \%$ below the expected Hall mobility. From this, it is clear that when examining slightly nonuniform samples, the FSS method is preferable to the F3S method.

We conclude that with a measurement time of less than a minute, the accuracy of the FSS method is reasonable for samples with sheet resistance variations below $5 \%$, whereas highly nonuniform samples must be characterized with the more time consuming AI method, which in turn does yield a Hall mobility with very high confidence, and with relative standard deviations of just $1 \%$ and $2 \%$, for the wafer with and without AL, respectively. However, the measurement time for the AI method is 30-40 minutes for one series of N, S, E, W measurements. The AI methods also validates the assumption of constant mobility, since it has been shown that the two pads have the same Hall mobility, while having significantly different sheet resistances.

1) Sheet carrier density: The sheet carrier density changes significantly towards the edges of the pads where the Hall mobilities are extracted. Thus, for a constant Hall mobility we find the activation degree of the implanted dose to be $14 \%$ and $21 \%$ in the pad centre and on average $18 \%$ and $20 \%$ at the pad edges for the wafer without and with AL, respectively. This is in reasonable good agreement with previously reported values for a non-patterned wafer [10].

\section{CONCLUSION}

Micro Hall Effect measurements have been performed on two different pads, one with a sheet resistance change toward the edge of $\approx-25 \%$ and the other with $\approx+5 \%$. Three different methods have been used on both samples to extract 
the Hall mobility. For both fast methods, it proved most difficult to extract correct values of Hall mobilities on the pad with the biggest change in sheet resistance. The proposed technique of measuring two points close to each other was shown to be more reliable than the existing method, where the two points were measured relatively far apart. Measuring two points close to each other resulted in an average Hall mobility that are around $10 \%$ too low, when measuring on the pad with the largest variation, while only a $2.3 \%$ wrong when measuring on the test pad with least sheet resistance variation. However, when combining detailed scanning of sheet resistance and micro Hall Effect, it has been demonstrated that Hall mobility can be determined with a relative standard deviation of 1-2 \%, even for highly non-uniform test pads.

\section{ACKNOWLEDGEMENTS}

We are grateful for the financial support from Copenhagen Graduate School for Nanoscience and Nanotechnology C:O:N:T, the Danish Research Agency FTP. Center for Individual Nanoparticle Functionality CINF is sponsored by The Danish National Research Foundation. We thank Peter F. Nielsen and Rong Lin for continuous help and support, and Peter Bøggild for encouragement and fruitful discussions.

\section{REFERENCES}

[1] D. K. Schroder, "Semiconductor materail and device characterization", 3rd ed. (Wiley, Hoboken, NJ, 2006).

[2] T. Clarysse, J. Bogdanowicz, J. Goossens, A. Moussa, E. Rosseel, W. Vandervorst, D. H. Petersen, R. Lin, P. F. Nielsen, O. Hansen, G. Merklin, N. S. Bennett, and N. E. B. Cowern, "On the analysis of the activation mechanisms of sub-melt laser anneals", Mater. Sci. and Eng. B, 154, 24 (2008).

[3] D. H. Petersen, O. Hansen, R. Lin and P. F. Nielsen , "Micro-four-point probe Hall effect measurement method", J. Appl. Phys. 104, 013710, 2008.

[4] D. H. Petersen, O. Hansen, P. F. Nielsen, T. Clarysse, J. Goossens, E. Rosseel and W. Vandervorst, "High precision micro-scale Hall Effect characterization mehod using in-line micro four-point probes", J. Vac. Sci. Technol. B, 26(1), 2008.

[5] F. W. Osterberg, D. H. Petersen, F. Wang, E. Rosseel, W. Vandervorst and $O$. Hansen, to be submitted.

[6] A. Hunter, J. Zelenko and R. Mani, Proc. IEEE RTP 2007, p.13.

[7] http://www.capres.com/

[8] S. Thorsteinsson, F. Wang, D. H. Petersen, T. M. Hansen, D. Kjær, R. Lin, J. Y. Kim, P. F. Nielsen and O. Hansen, "Accurate micro four-point probe sheet resistance measurements on small samples", Review of Scientific Instruments, Vol. 80, Issue 5, pp. 053902-053902-10, 2009

[9] E. Rosseel, D. H. Petersen, F. W. Osterberg, O. Hansen, J. Bogdanowicz, T. Clarysse, W. Vandervorst, C. Ortolland, T. Hoffmann, P. Chan, A. Salnik and L. Nicolaides, "Monitoring of local and global temperature non-uniformities by means of Therma-Probe and Micro Four-Point Probe metrology", Proc. IEEE RTP 2009.

[10] E. Rosseel, W. Vandervorst, T. Clarysse, J. Goossens, A. Moussa, R. Lin, D. H. Petersen, P. F. Nielsen, O. Hansen, N. S. Bennett, and N. E. B. Cowern, "Impact of multiple sub-melt laser scans on the activation and diffusion of shallow Boron junctions", Proc. IEEE RTP 2008. 